\title{
Real-Valued Khatri-Rao Subspace Approaches on the ULA and a New Nested Array
}

\author{
Huiping Duan, Tiantian Tuo, Jun Fang and Bing Zeng
}

\begin{abstract}
In underdetermined direction-of-arrival (DOA) estimation using the covariance-based signal models, the computational complexity turns into a noticeable issue because of the high dimension of the virtual array manifold. In this paper, real-valued Khatri-Rao (KR) approaches are developed on the uniform linear array (ULA) and the nested array. The complexities of subspace decomposition and spectral search are reduced compared with the complex-valued KR approach. By designing a special transformation matrix, the influence of the noise is removed in the mean time while the data is transformed from the complex domain to the real domain. Deploying the sensors with nonuniform spacings can raise the degree of freedom (DOF) and hence help detect more sources in the underdetermined situation. To increase the DOF further, a new nested array geometry is designed. The real-valued denoising KR approach developed on the new nested array can resolve more sources with reduced complexities. The performance improvement is demonstrated by numerical studies.
\end{abstract}

\section{Index Terms}

underdetermined direction-of-arrival (DOA) estimation, Khatri-Rao product, real-valued, nested array, degree of freedom (DOF)

Huiping Duan, Tiantian Tuo and Bing Zeng are with the School of Electronic Engineering, University of Electronic Science and Technology of China, Chengdu, China, 611731, Email: huipingduan@uestc.edu.cn, 201321020471@std.uestc.edu.cn, eezeng@uest.edu.cn.

Jun Fang is with the National Key Laboratory of Science and Technology on Communications, University of Electronic Science and Technology of China, Chengdu, China, 611731, Email: JunFang@uestc.edu.cn.

This work was supported in part by the National Natural Science Foundation of China under Grants 61201274, 61172114, 61370148. 


\section{INTRODUCTION}

In source localization using antenna arrays in radar, sonar and communication systems, the underdetermined situation [1]-[3], where the number of sources exceeds the number of sensors, has been paid special attentions.

In order to detect more sources, covariance-based algorithms have been explored. In these algorithms, the degree of freedom (DOF), which is measured by the number of distinct cross correlation terms in the associated difference co-array [4], plays an important role in determining the number of sources the array can identify. In [5], the Khatri-Rao (KR) subspace approach is developed. The DOF of an N-element uniform linear array (ULA) is increased to $2 N-1$ by exploiting the self-Khatri-Rao product structure of the array manifold matrix and $2 N-2$ sources can be identified with the KR-MUSIC algorithm. The computational complexity turns into a noticeable issue because of the high dimension of the virtual array manifold. Although a dimension reduction strategy is adopted in [5], subspace decomposition and spectral search are still computationally expensive due to the complex-valued operations in the algorithm.

Deploying the sensors with nonuniform spacings can raise the DOF and hence help the covariancebased algorithms detect more sources in the underdetermined situation. Nonuniform linear arrays, like the minimum redundancy array (MRA) [6] and the non-redundant array (NRA) [2], have been designed to enhance the DOF. However, no general analytical formulations can be provided to express the array geometry or the DOF due to the lack of regularity in sensor deployment in MRA and NRA. Lately, two types of nonuniform linear arrays, the co-prime array and the nested array, are proposed in [4], [7]. The nested array consists of two or more ULAs with increasing intersensor spacings. The DOF achieved by using an N-element nested array is $\mathcal{O}\left(N^{2}\right)$. Compared with MRA and NRA, the uniform geometry inside each level of the nested array simplifies the formulation and analysis but sacrifices the DOF. It is desirable to achieve higher DOF by inerratic array geometry.

In this paper, real-valued KR approaches are developed for the underdetermined direction-of-arrival (DOA) estimation problem. The complexities of subspace decomposition and spectral search are reduced compared with the complex-valued KR approach. By designing a special transformation matrix, the influence of the noise is removed in the mean time while the data is transformed from the complex domain to the real domain. Unlike using the orthogonal complement projecting [5] or eliminating entries of the covariance matrix by additional matrix multiplication [8], no extra operations are required for eliminating the noise in the proposed real-valued KR approaches. By relocating the origin of coordinate and increasing the sensor spacing of the outer-level ULA of Pal's nested array, a new nested array 
geometry is designed. The real-valued denoising KR approach developed on the new nested array can resolve more sources with reduced computational complexity and increased spectral search efficiency.

The rest of the paper is organized as follows: the signal model based on the KR product is described in Section III In Section [II] the real-valued KR approach on the ULA is designed. The new nested array geometry and the real-valued KR approach developed on it are presented in Section IV. Simulation results are demonstrated in Section $\mathrm{V}$. Finally, the paper is concluded in Section VI.

\section{Signal Model BASED on the KR PROdUCT}

Consider $K$ narrowband far-field signals impinging on an N-element uniform or non-uniform linear array. The signals are assumed to be zero-mean quasi-stationary sources [5] with locally static secondorder statistics. The array received data at $T$ time snapshots is modeled as

$$
\mathbf{x}(t)=\mathbf{A} \mathbf{s}(t)+\mathbf{v}(t), \quad t=0,1, \ldots, T-1
$$

with $\mathbf{x}(t)=\left[x_{1}(t) \cdots x_{N}(t)\right]^{T}$ and $\mathbf{s}(t)=\left[s_{1}(t) \cdots s_{K}(t)\right]^{T} . x_{n}(t)$ is the data received by the $n$th sensor. $s_{k}(t), k=1,2, \ldots, K$ are the sources which are uncorrelated with each other. $\mathbf{v}(t) \in \mathbb{C}^{N \times 1}$ is the noise assumed to be zero-mean wide-sense stationary and statistically independent of the source signals. $\mathbf{A}=\left[\begin{array}{lll}\mathbf{a}\left(\theta_{1}\right) & \cdots & \mathbf{a}\left(\theta_{k}\right)\end{array}\right] \in \mathbb{C}^{N \times K}$ is the array manifold matrix where $\theta_{k} \in[-\pi / 2, \pi / 2]$ is the DOA of the $k$ th source, and

$$
\mathbf{a}\left(\theta_{k}\right)=\left[\begin{array}{lll}
e^{-\frac{j 2 \pi d_{1}}{\lambda} \sin \theta_{k}} & \cdots & e^{-\frac{j 2 \pi d_{N}}{\lambda} \sin \theta_{k}}
\end{array}\right]^{T}
$$

is the $k$ th steering vector. Here, $\lambda$ and $d_{i}$ represent the signal wavelength and the location of the $i$ th sensor, respectively.

Divide the $T$ snapshots into frames with the frame length $L$. The local covariance matrix is defined as

$$
\mathbf{R}_{m}=\mathrm{E}\left\{\mathbf{x}(t) \mathbf{x}^{H}(t)\right\}, \forall t \in[(m-1) L, m L-1],
$$

where $m \in[1, M]$ represents the frame index. $\mathbf{R}_{m}$ can be formulated as

$$
\begin{aligned}
\mathbf{R}_{m} & =\mathbf{A D}_{m} \mathbf{A}^{H}+\mathbf{C} \\
& =\mathbf{A}\left[\begin{array}{cccc}
\sigma_{m 1} & 0 & \cdots & 0 \\
0 & \sigma_{m 2} & \cdots & 0 \\
\vdots & \vdots & \ddots & \vdots \\
0 & 0 & \cdots & \sigma_{m K}
\end{array}\right] \mathbf{A}^{H}+\mathbf{C},
\end{aligned}
$$

where $\sigma_{m k}=\mathrm{E}\left\{\left|s_{k}(t)\right|^{2}\right\}$ is the source power, and $\mathbf{C}=\mathbf{E}\left\{\mathbf{v}(\mathbf{t}) \mathbf{v}^{\mathbf{H}}(\mathbf{t})\right\}$ is the noise covariance matrix. 
Following [5], $\mathbf{R}_{m}$ is vectorized:

$$
\begin{aligned}
\mathbf{y}_{m} & =\operatorname{vec}\left(\mathbf{R}_{m}\right)=\operatorname{vec}\left(\mathbf{A D}_{m} \mathbf{A}^{H}\right)+\operatorname{vec}(\mathbf{C}) \\
& =\left(\mathbf{A}^{*} \odot \mathbf{A}\right) \mathbf{d}_{m}+\operatorname{vec}(\mathbf{C}),
\end{aligned}
$$

where $\odot$ represents the Khatri-Rao product:

$$
\mathbf{A}^{*} \odot \mathbf{A}=\left[\mathbf{a}^{*}\left(\theta_{1}\right) \otimes \mathbf{a}\left(\theta_{1}\right) \cdots \mathbf{a}^{*}\left(\theta_{k}\right) \otimes \mathbf{a}\left(\theta_{k}\right)\right]
$$

with $\bigotimes$ denoting the Kronecker product and $\mathbf{d}_{m}=\operatorname{Diag}\left\{\mathbf{D}_{m}\right\}$. Letting $\mathbf{Y}=\left[\begin{array}{lll}\mathbf{y}_{1} & \cdots & \mathbf{y}_{M}\end{array}\right]$, we have

$$
\mathbf{Y}=\left(\mathbf{A}^{*} \odot \mathbf{A}\right) \mathbf{\Psi}^{T}+\operatorname{vec}(\mathbf{C}) \mathbf{1}_{M}^{T},
$$

where $\mathbf{1}_{M}=\left[\begin{array}{lll}1 & \cdots & 1\end{array}\right]^{T} \in R^{M}$ and

$$
\begin{aligned}
\boldsymbol{\Psi} & =\left[\begin{array}{lll}
\mathbf{d}_{1} & \cdots & \mathbf{d}_{M}
\end{array}\right]^{T} \\
& =\left[\begin{array}{cccc}
d_{11} & d_{12} & \cdots & d_{1 K} \\
d_{21} & d_{22} & \cdots & d_{2 K} \\
\vdots & \vdots & \ddots & \vdots \\
d_{M 1} & d_{M 2} & \cdots & d_{M K}
\end{array}\right] .
\end{aligned}
$$

Compared with (1), $\mathbf{Y}$ in (7) is just like the data received at an array whose manifold matrix is $\mathbf{A}^{*} \odot \mathbf{A}$. Hence, instead of (1), DOA estimation can be carried out based on the Khatr-Rao-product model (7). According to [5], for quasi-stationary sources with long enough sampling duration and sufficient power variation, $\operatorname{rank}(\Psi)=\mathbf{K}$ can be satisfied. $\mathbf{A}^{*} \odot \mathbf{A}$ is full column rank When $K \leq 2 N-1$.

\section{ReAL-VALUED KR APPROACH ON THE ULA}

Consider a linear array with the uniform geometry. Applying the dimension-reduction idea in [5], the virtual array manifold can be written as

$$
\mathbf{A}^{*} \odot \mathbf{A}=\mathbf{G} \widetilde{\mathbf{B}}
$$

where $\mathbf{G}=\left[\begin{array}{llll}\mathbf{G}_{1}^{T} & \mathbf{G}_{2}^{T} & \cdots & \mathbf{G}_{N}^{T}\end{array}\right]^{T} \in \mathbb{C}^{N^{2} \times(2 N-1)}$ with all entries of the matrix $\mathbf{G}_{i} \in \mathbb{C}^{N \times(2 N-1)}$ being zero except

$$
\mathbf{G}_{i}(1: N, N+1-i: 2 N-i)=\mathbf{I}_{N}, i=1,2, \ldots, N
$$

where $\mathbf{I}_{N}$ represents the $N \times N$ identity matrix. $\widetilde{\mathbf{B}}=\left[\mathbf{b}\left(\theta_{1}\right), \ldots, \mathbf{b}\left(\theta_{K}\right)\right] \in \mathbb{C}^{(2 N-1) \times K}$ with

$$
\begin{aligned}
& \mathbf{b}(\theta)=\left[\begin{array}{lllll}
e^{(N-1) \frac{j 2 \pi d}{\lambda} \sin \theta} & \cdots & e^{\frac{j 2 \pi d}{\lambda} \sin \theta} & 1 \cdots
\end{array}\right. \\
& \left.e^{-(N-1) \frac{j 2 \pi d}{\lambda} \sin \theta}\right] \text {. }
\end{aligned}
$$


Let $\mathbf{W}=\mathbf{G}^{T} \mathbf{G}$. It can be derived that

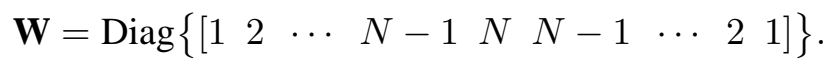

The dimension of $\mathbf{Y}$ in (7) can be reduced by a linear transformation:

$$
\begin{aligned}
\tilde{\mathbf{Y}} & =\mathbf{W}^{-1 / 2} \mathbf{G}^{T} \mathbf{Y} \\
& =\mathbf{W}^{1 / 2} \tilde{\mathbf{B}} \Psi^{T}+\mathbf{W}^{-1 / 2} \mathbf{G}^{T} \operatorname{vec}(\mathbf{C}) \mathbf{1}_{M}^{T} .
\end{aligned}
$$

In fact, by left multiplying $\mathbf{W}^{-\mathbf{1} / \mathbf{2}} \mathbf{G}^{\mathbf{T}}$ on $\mathbf{Y}$, the repeated rows of $\mathbf{A}^{*} \odot \mathbf{A}$ are averaged and sorted. Let $\mathbf{B}=\mathbf{W}^{1 / 2} \tilde{\mathbf{B}}$ be the virtual array manifold after dimension reduction. To separate the real and the imaginary parts, (13) is rewritten as

$$
\begin{aligned}
& \tilde{\mathbf{Y}}_{R}+j \tilde{\mathbf{Y}}_{I}=\left(\mathbf{B}_{R}+j \mathbf{B}_{I}\right) \mathbf{\Psi}^{T}+ \\
& \mathbf{W}^{-1 / 2} \mathbf{G}^{T} \operatorname{vec}(\mathbf{C}) \mathbf{1}_{M}^{T}
\end{aligned}
$$

Define two matrices $\mathbf{H}_{1} \in \mathbb{R}^{(N-1) \times(2 N-1)}$ and $\mathbf{H}_{2} \in \mathbb{R}^{(N-1) \times(2 N-1)}$ as follows:

$$
\begin{aligned}
& \mathbf{H}_{1}=\frac{1}{\sqrt{2}}\left[\begin{array}{ccccccc}
1 & \cdots & 0 & 0 & 0 & \cdots & 1 \\
\vdots & \ddots & \vdots & \vdots & \vdots & . \cdot & \vdots \\
0 & \cdots & 1 & 0 & \underbrace{1}_{N-1} & \cdots & 0
\end{array}\right], \\
& \mathbf{H}_{2}=\frac{1}{\sqrt{2} j}\left[\begin{array}{ccccccc}
1 & \cdots & 0 & 0 & 0 & \cdots & -1 \\
\vdots & \ddots & \vdots & \vdots & \vdots & . \cdot & \vdots \\
0 & \cdots & 1 & 0 & \underbrace{-1}_{N-1} & \cdots & 0 \\
N-1
\end{array}\right] \text {. }
\end{aligned}
$$

Multiply $\mathbf{H}_{1}$ and $\mathbf{H}_{2}$ on the left side of equation (14):

$$
\begin{aligned}
& \mathbf{H}_{1} \tilde{\mathbf{Y}}=\mathbf{H}_{1} \mathbf{B} \Psi^{T}+\mathbf{H}_{1} \mathbf{W}^{-1 / 2} \mathbf{G}^{T} \operatorname{vec}(\mathbf{C}) \mathbf{1}_{M}^{T}, \\
& \mathbf{H}_{2} \tilde{\mathbf{Y}}=\mathbf{H}_{2} \mathbf{B} \Psi^{T}+\mathbf{H}_{2} \mathbf{W}^{-1 / 2} \mathbf{G}^{T} \operatorname{vec}(\mathbf{C}) \mathbf{1}_{M}^{T} .
\end{aligned}
$$

As we can see, $\mathbf{H}_{1} \mathbf{B}=\mathbf{B}_{R}$ and $\mathbf{H}_{2} \mathbf{B}=\mathbf{B}_{I}$ are the real part and the imaginary part of $\mathbf{B}$ multiplied by a scaling coefficient $\sqrt{2}$, respectively.

For spatially uniform or nonuniform white noise with covariance matrix $\left.\mathbf{C}=\operatorname{Diag}\left\{\begin{array}{llll}\sigma_{\mathbf{n} 1}^{2} & \sigma_{\mathbf{n} 2}^{2} & \cdots & \sigma_{\mathbf{n N}}^{2}\end{array}\right]\right\}$, we can derive that $\mathbf{W}^{-1 / 2} \mathbf{G}^{T} \operatorname{vec}(\mathbf{C})=\frac{1}{\sqrt{N}} \sum_{i=1}^{N} \sigma_{n i}^{2} \mathbf{e}=\sigma_{\mathbf{n}}^{\mathbf{2}} \mathbf{e}$, where $\mathbf{e}$ is an $N^{2} \times 1$ vector with the Nth element being one and all other elements being zero. Since the Nth columns of $\mathbf{H}_{\mathbf{1}}$ and $\mathbf{H}_{\mathbf{2}}$ are all-zero vectors, by simple mathematical operations, we can prove that

$$
\begin{aligned}
& \mathbf{H}_{1} \mathbf{W}^{-1 / 2} \mathbf{G}^{T} \operatorname{vec}(\mathbf{C}) \mathbf{1}_{M}^{T}=\mathbf{0}, \\
& \mathbf{H}_{2} \mathbf{W}^{-1 / 2} \mathbf{G}^{T} \operatorname{vec}(\mathbf{C}) \mathbf{1}_{M}^{T}=\mathbf{0} .
\end{aligned}
$$


Hence, with the transformation matrices $\mathbf{H}_{1}$ and $\mathbf{H}_{2}$, the additive noise is eliminated and (16) can be rewritten as

$$
\overline{\mathbf{Y}}=\left[\begin{array}{c}
\mathbf{H}_{1} \\
\mathbf{H}_{2}
\end{array}\right] \tilde{\mathbf{Y}}=\mathbf{H} \tilde{\mathbf{Y}}=\left[\begin{array}{c}
\mathbf{B}_{R} \\
\mathbf{B}_{I}
\end{array}\right] \mathbf{\Psi}^{\mathbf{T}} .
$$

Due to the all-zero column in $\mathbf{H}$, left multiplication of $\mathbf{H W}^{-\mathbf{1} / \mathbf{2}} \mathbf{G}^{\mathbf{T}}$ lead to the loss of one degree of freedom and hence $K \leq 2 N-2$ has to be satisfied. We perform the singular value decomposition (SVD) on $\overline{\mathbf{Y}}$ :

$$
\overline{\mathbf{Y}}=\mathbf{U} \boldsymbol{\Sigma} \mathbf{V}^{\mathbf{H}}
$$

where $\mathbf{U} \in \mathbb{C}^{(2 N-2) \times(2 N-2)}$ and $\mathbf{V} \in \mathbb{C}^{M \times M}$ are the left and right singular matrices, respectively, and $\boldsymbol{\Sigma} \in \mathbb{R}^{(\mathbf{2 N}-\mathbf{2}) \times \mathbf{M}}$ contains the singular values in descending order. Then the noise subspace is estimated as:

$$
\mathbf{U}_{n}=\left[\mathbf{u}_{K+1}, \ldots, \mathbf{u}_{2 N-2}\right] \in \mathbb{C}^{(2 N-2) \times(2 N-2-K)} .
$$

The dimension of $\mathbf{U}_{n}$ further limits $K$ to be less than $2 N-2$. Finally the spatial spectrum can be calculated as follows:

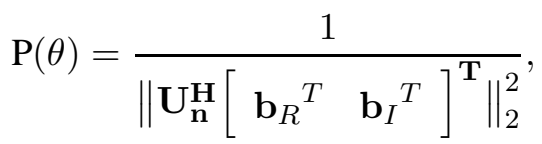

where $\mathbf{b}_{\mathbf{R}}=\mathbf{H}_{\mathbf{1}} \mathbf{W}^{\mathbf{1} / \mathbf{2}} \mathbf{b}(\theta)$ and $\mathbf{b}_{\mathbf{I}}=\mathbf{H}_{\mathbf{2}} \mathbf{W}^{\mathbf{1} / \mathbf{2}} \mathbf{b}(\theta)$.

\section{Real-VAlued KR Approach on the Nested ARray}

A new nested array geometry is designed before developing the real-valued Khatri-Rao subspace approach on it.

\section{A. The New Nested Geometry}

Similar to Pal's array [4], the proposed nested array consists of two concatenated ULAs which are called the inner and the outer. The inner ULA has $N_{1}$ elements with spacing $d_{1}$ and the outer ULA has $N_{2}$ elements with spacing $d_{2}$. The Pal's nested array sets $d_{2}=\left(N_{1}+1\right) d_{1}$ while the new geometry sets $d_{2}=N_{1} d_{1}$. Note that the new geometry puts the origin of coordinate on the first sensor of the inner level. More specifically, the sensors' locations of the new nested array are given by $S_{\text {inner }}=\left\{(m-1) d_{1}, m=\right.$ $\left.1,2, \ldots, N_{1}\right\}$ and $S_{\text {outer }}=\left\{(n+1) N_{1} d_{1}, n=1,2, \ldots, N_{2}\right\}$. According to the knowledge of the difference set, if $N_{2} \geq 2$, this nested array is equivalent to a filled ULA with $2\left(N_{2}+1\right) N_{1}+1$ elements whose positions are given by

$$
S_{c a}=\left\{n d_{1}, n=-\tilde{M}, \ldots, \tilde{M}, \tilde{M}=\left(N_{2}+1\right) N_{1}\right\} .
$$




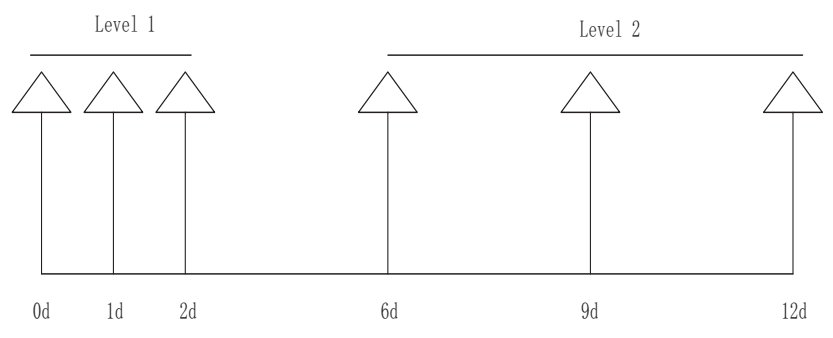

Fig. 1. The proposed two-level nested array with 3 sensors in each level.

TABLE I: Comparison of the DOF.

\begin{tabular}{c|c|c|c|c|c}
\hline$N_{1}+N_{2}$ & MRA & Coprime array & ULA (KR) & Pal's & Proposed \\
\hline $3+2$ & 19 & 6 & 9 & 15 & 19 \\
$5+2$ & 35 & 10 & 13 & 23 & 31 \\
$5+3$ & 47 & 15 & 15 & 35 & 41 \\
$7+3$ & 73 & 21 & 19 & 47 & 57 \\
\hline Formula & inexistent & $N_{1} \times N_{2}$ & $2\left(N_{1}+N_{2}\right)-1$ & $2 N_{2}\left(N_{1}+1\right)-1$ & $2\left(N_{2}+1\right) N_{1}+1$ \\
\hline
\end{tabular}

Figure 1 shows an example of this new nested array geometry with $N_{1}=N_{2}=3$. As comparisons, in Table I] the DOF obtained by the MRA [6], the coprime array [7], the ULA (in the KR subspace method [5]), the Pal's two-level nested array [4] and the proposed two-level nested array are listed for different values of $N_{1}$ and $N_{2}$. The new two-level nested array can attain $2\left(N_{2}+1\right) N_{1}+1$ DOF using $N_{1}+N_{2}$ elements. When $N_{1} \geq N_{2}$, the new nested array can increase up to $2\left(N_{1}-N_{2}\right)+2$ DOF compared with Pal's nested array.

\section{B. Real-Valued KR Approach on the Nested Array}

Consider model (7) on the new nested array with $N_{1}+N_{2}$ sensors. The dimension of the virtual array manifold $\mathbf{A}^{*} \odot \mathbf{A}$ is $\left(N_{1}+N_{2}\right)^{2} \times K$. It has $2\left(N_{2}+1\right) N_{1}+1$ distinct rows which is as many as the DOF of the nested array. As what we do for the ULA, the virtual array manifold $\mathbf{A}_{1} \in \mathbb{C}^{2\left(N_{2}+1\right) N_{1}+1}$ is constructed from $\mathbf{A}^{*} \odot \mathbf{A}$ by averaging the repeated rows and sorting the elements so that the $i$ th row of the matrix corresponds to the $-\left(N_{2}+1\right) N_{1}+i$ th position of the virtual array. Although it is hard to provide a general expression for $\mathbf{G}$ and $\mathbf{W}$ for the nested array with the arbitrarily given number of sensors, the operations of averaging and sorting can be executed on the observation matrix $\mathbf{Y}$ in (7) to 
obtain a new matrix $\mathbf{Z}$ as follows

$$
\mathbf{Z}=\mathbf{A}_{1} \Psi^{T}+\sigma_{n}^{2} \hat{\mathbf{e}}
$$

where $\hat{\mathbf{e}} \in \mathbb{R}^{\left(2\left(N_{2}+1\right) N_{1}+1\right) \times M}$ is a matrix whose elements are all-zero except that the $\left(N_{2}+1\right) N_{1}+1$ th row is an all-one vector.

Two matrices, $\hat{\mathbf{H}}_{1} \in R^{\left(N_{2}+1\right) N_{1} \times\left(2\left(N_{2}+1\right) N_{1}+1\right)}$ and $\hat{\mathbf{H}}_{2} \in R^{\left(N_{2}+1\right) N_{1} \times\left(2\left(N_{2}+1\right) N_{1}+1\right)}$, are defined to transform the complex-valued data into the real-valued one:

$$
\begin{aligned}
& \hat{\mathbf{H}}_{1}=\frac{1}{\sqrt{2}}\left[\begin{array}{ccccccc}
1 & \cdots & 0 & 0 & 0 & \cdots & 1 \\
\vdots & \ddots & \vdots & \vdots & \vdots & . & \vdots \\
\underbrace{0}_{\left(N_{2}+1\right) N_{1}} & \cdots & 1 & 0 & \underbrace{1}_{\left(N_{2}+1\right) N_{1}} & \cdots & 0
\end{array}\right], \\
& \hat{\mathbf{H}}_{2}=\frac{1}{\sqrt{2} j}\left[\begin{array}{ccccccc}
1 & \cdots & 0 & 0 & 0 & \cdots & -1 \\
\vdots & \ddots & \vdots & \vdots & \vdots & . & \vdots \\
0 & \cdots & 1 & 0 & \underbrace{-1}_{\left(N_{2}+1\right) N_{1}} & \cdots & 0 \\
\left(N_{2}+1\right) N_{1}
\end{array}\right] \text {. }
\end{aligned}
$$

The transformations are as follows:

$$
\begin{aligned}
\hat{\mathbf{H}}_{1} \mathbf{Z} & =\hat{\mathbf{H}}_{1} \mathbf{A}_{1} \boldsymbol{\Psi}^{T}+\hat{\mathbf{H}}_{1} \sigma_{n}^{2} \hat{\mathbf{e}} \\
\hat{\mathbf{H}}_{2} \mathbf{Z} & =\hat{\mathbf{H}}_{2} \mathbf{A}_{1} \boldsymbol{\Psi}^{T}+\hat{\mathbf{H}}_{2} \sigma_{n}^{2} \hat{\mathbf{e}}
\end{aligned}
$$

Since $\hat{\mathbf{H}}_{1} \hat{\mathbf{e}}=\mathbf{0}$ and $\hat{\mathbf{H}}_{2} \hat{\mathbf{e}}=\mathbf{0}$, the influence of the noise is eliminated. Let $\mathbf{A}_{1 R}=\hat{\mathbf{H}}_{1} \mathbf{A}_{1}$ and $\mathbf{A}_{1 I}=$ $\hat{\mathbf{H}}_{2} \mathbf{A}_{1}$ be the real and the imaginary parts of the virtual array response matrices. The new model is formulated for the nested array:

$$
\overline{\mathbf{Z}}=\left[\begin{array}{c}
\hat{\mathbf{H}}_{1} \mathbf{Z} \\
\hat{\mathbf{H}}_{2} \mathbf{Z}
\end{array}\right]=\left[\begin{array}{c}
\mathbf{A}_{1 R} \\
\mathbf{A}_{1 I}
\end{array}\right] \mathbf{\Psi}^{T} .
$$

Then SVD can be performed on $\overline{\mathbf{Z}}$ to get the noise subspace which is applied to search the spectral peaks.

\section{NumericAl STUdies}

Numerical studies are carried out to demonstrate the performance of the proposed real-valued KR approaches on the ULA and the new nested array. We consider a ULA with 6 sensors $(N=6)$ and a 2-level nested array with 3 sensors in each level $\left(N_{1}=3, N_{2}=3\right)$. The quasi-stationary sources with uniformly distributed random frame lengths are simulated. The array snapshots are divided into $M$ frames 


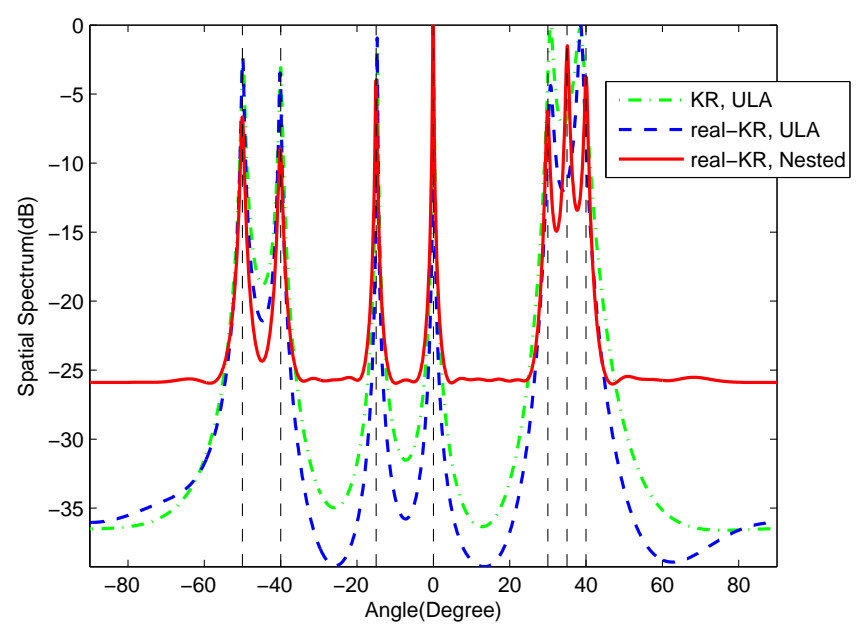

Fig. 2. Spatial spectra in an underdetermined situation.

with the frame length $L$ to estimate the local covariance matrices. The spatial noise is zero-mean and uniformly white complex Gaussian.

A narrowband underdetermined case with 7 sources from different directions, $\left\{-50^{\circ},-40^{\circ},-15^{\circ}, 0^{\circ}, 30^{\circ}\right.$, $35^{\circ}, 40^{\circ}$, is studied. The signal to noise ratio (SNR) is set as OdB. Simulations use a total of 20000 snapshots $(T)$ with 400 snapshots $(L)$ in each of the 50 frame intervals $(M)$. The spatial spectra obtained by the KR subspace method and the real-valued KR approaches on the ULA and the new nested array are plotted in Figure 2. We can see that the complex-valued and the real-valued KR approaches on the ULA provide similar spatial spectra. The new nested array shows satisfactory resolving capability while the ULA fails in resolving the closely spaced sources in this case.

The root mean square error (RMSE) is evaluated by 1000 Monte Carlo simulations. One source from $15^{\circ}$ is assumed and the SNR varies from $-10 \mathrm{~dB}$ to $14 \mathrm{~dB}$. As shown in Figure 3, the new nested array exhibits significantly lower RMSE than the ULA. It is observed that the real-valued KR subspace method performs better than the complex-valued KR approach at moderate or low SNR situations.

In addition, the average time spent in performing SVD and searching spectral peaks over 100 trials are listed in Table $\amalg$, The real-valued KR approaches on both the ULA and the new nested array achieve reduced computational complexity and increased searching efficiency. This can be explained by the fact that a complex multiplication is completed by four real multiplications and two real additions, and a complex addition is completed by two real additions. 


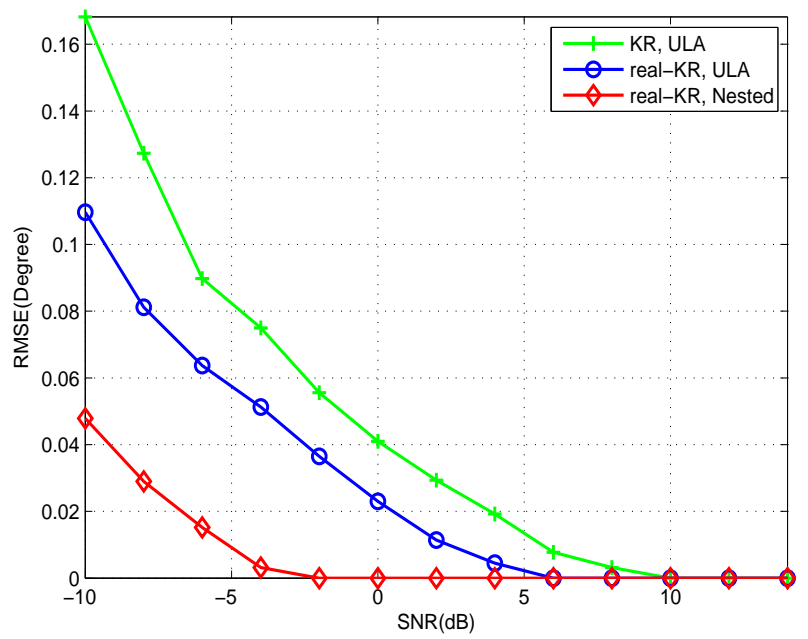

Fig. 3. RMSE vs SNR.

TABLE II: SVD Calculation Time and Spectral Search Time (millisecond).

\begin{tabular}{l|c|c}
\hline Algorithm & SVD calculation time & Spectral search time \\
\hline KR, ULA & 0.321 & 6.0 \\
real-KR, ULA & 0.152 & 3.3 \\
KR, Nested & 0.678 & 7.4 \\
real-KR, Nested & 0.621 & 4.7 \\
\hline
\end{tabular}

\section{CONCLUSIONS}

In this paper, the underdetermined DOA estimation problem is studied by developing real-valued KR subspace methods and a nonuniform array geometry. The new nested array geometry can increase the DOF from $2 N_{2}\left(N_{1}+1\right)-1$ to $2\left(N_{2}+1\right) N_{1}+1$. Here, $N_{1}$ and $N_{2}$ are the numbers of sensors in the inner and outer levels of the nested array. The real-valued denoising KR approach developed on the new nested array can resolve more sources with reduced computational complexity.

\section{REFERENCES}

[1] H. L. V. Trees, Detection, Estimation, and Modulation Theory, Part IV, Optimum Array Processing, New York: Wiley, 2002.

[2] S. Haykin, J. P. Reilly, V. Kezys, and E. Vertatschitsch, "Some aspects of array signal processing," IEE Proceedings-F, vol. 139, no. 1, pp. 1-26, Feb. 1992. 
[3] Z.-Q. He, Z.-P. Shi, L. Huang, and H. C. So, "Underdetermined DOA estimation for wideband signals using robust sparse covariance fitting," IEEE Signal Processing Lett., vol. 22, no. 4, pp. 435-439, Apr. 2015.

[4] P. Pal and P. P. Vaidyanathan, "Nested arrays: A novel approach to array processing with enhanced degrees of freedom," IEEE Trans. Antennas Propagat., vol. 58, no. 8, pp. 4167-4181, Aug. 2010.

[5] W.-K. Ma, T.-H. Hsieh, and C.-Y. Chi, "DOA estimation of quasi-stationary signals with less sensors than sources and unknown spatial noise covariance: A Khatri-Rao subspace approach,” IEEE Trans. Antennas Propagat., vol. 58, no. 4, pp. 2168-2180, Apr. 2010.

[6] T. A. Moffet, "Minimum redundancy linear arrays," IEEE Trans. Antennas Propagat., vol. 16, no. 2, pp. 172-175, Mar. 1968.

[7] P. Pal and P. P. Vaidyanathan, "Sparse sensing with co-prime samplers and arrays," IEEE Trans. Signal Processing, vol. 59, no. 2, pp. 573-586, Feb. 2011.

[8] Z.-Q. He, Z.-P. Shi, and L. Huang, "Covariance sparsity-aware DOA estimation for nonuniform noise," Digital Signal Processing, vol. 28, 2014. 